tumours which were previously considered to be untreatable. This also leads to an important issue: as cancer therapy becomes more effective, it is important to understand the mechanisms behind serious adverse drug reactions associated with anti-cancer drugs in order to further improve the benefit-risk ratio, and reduce cancer survivor issues. The identification of a genetic predisposing factor for an adverse drug reaction may have several benefits: (a) a better understanding of the mechanism of toxicity, which may allow the development of interventional agents, but will also feed into future drug development; and (b) the development of predictive testing which could then be used prior to drug prescription to optimise both the choice and dose of drug. Both these scenarios require the identification and recruitment of deeply phenotyped patients, a major limitation at present. For predictive testing, a validated genetic test is required together with an appropriate interventional strategy to reduce harm (increased monitoring, alteration of dose or different drug choice).

\section{BCR-ABL1 AND BEYOND}

$\underline{\text { Susan Branford }}$

Centre for Cancer Biology, SA Pathology, Adelaide, SA, Australia

Chronic myeloid leukaemia (CML) is characterised by the BCRABL1 fusion, which causes activated and abnormal cell signalling. If untreated, CML progresses to a rapidly fatal acute leukaemia at a median of 3 years. BCR-ABL1 can be superbly targeted by tyrosine kinase inhibitor drugs and most patients now have a life expectancy approaching that of the general population. However, about $20 \%$ of patients fail therapy and require a rapid change of treatment to avoid the risk of disease progression and death. Molecular monitoring of BCR-ABL1 transcript levels using quantitative PCR is now the recommended monitoring strategy and can detect treatment failure and emerging drug resistance. Acquired resistance is most frequently associated with mutations in the BCR-ABL1 kinase domain that interfere with drug binding. These are detected in $\sim 50 \%$ of resistant patients, but the other resistance mechanisms are not well understood. Data is emerging from next-generation sequencing studies that somatic variants in cancer-related genes occur at a high frequency at drug resistance. Furthermore, these mutations have also been detected in some patients at diagnosis and are associated with a poor outcome. Next-generation sequencing may provide important prognostic information for CML patients at diagnosis and resistance and identify targets for therapy.

\section{PREVENTING ADVERSE DRUG REACTIONS}

Munir Pirmohamed

MRC Centre for Drug Safety Science and Wolfson Centre for Personalised Medicine, The University of Liverpool, Liverpool, $U K$

Adverse drug reactions (ADRs) are a major clinical problem accounting for a great deal of morbidity, mortality and are a drain on healthcare resources. ADRs can generally be divided into ontarget and off-target reactions. Both types of ADRs have a genetic predisposition, but the quantitative contribution of genetic vs non-genetic factors varies with the type of reaction, the drug implicated and the patient's clinical co-morbidities. My talk will focus on genetic factors predisposing to ADRs, and how advances over the last 20 years have led not only to discovery, but also to some genetic tests becoming incorporated into clinical practice. There are some well-known polymorphisms in genes such as Glucose-6-phosphate dehydrogenase and butyrylcholinesterase which have been known about for decades, and testing can be undertaken in healthcare systems. More recently, the role of HLA and predisposition to immune mediated adverse reactions has been particularly fertile in identifying new associations, often through genome wide technologies. Indeed, since 2001, at least 30 new HLA-ADR associations have been reported. Two of these are in clinical practice $(H L A-B * 57: 01$ for abacavir hypersensitivity, and $H L A-B * 15: 02$ for carbamazepineinduced Stevens-Johnson Syndrome). Genetic factors can also determine dose; for example, for warfarin, polymorphisms in $V K O R C 1$ and $C Y P 2 C 9$ account for almost $50 \%$ of the variance in individual dose requirements. Investigation of the genomic basis of ADRs is not only important for development of predictive genetic testing, but can also provide insights into the mechanisms of ADRs.

\section{IS THERE A PLACE FOR POLYGENIC RISK SCORES AND GWAS IN MODERN GENETIC TESTING?}

\section{$\underline{\text { Stuart MacGregor }}$ \\ QIMR Berghofer Medical Research Institute, Brisbane, Qld, Australia}

Traditionally genetic testing has focused on rare high penetrance mutations such as BRCAl mutations conferring risk of breast/ ovarian cancer or $M Y O C$ mutations conferring risk of glaucoma. However, most Australians affected by common diseases such as these do not carry these mutations. Instead, most people are at high genetic risk due to the cumulative effect of many polygenes (that is, variants of individually small effect on risk).

Recent genome-wide association studies have identified polygenes for virtually every complex human trait. There has been considerable recent excitement about polygenic risk scores (PRS) - these provide an index of genetic risk based on the cumulative effect of many polygenes. While there are now PRS for many diseases, their translational utility can be limited due to poor predictive performance or because of a lack of real world consequences.

In my talk I will discuss applications of PRS across a range of traits including eye disease and cancer. In a general population setting I will show there are exciting opportunities for PRS to be used in improving risk stratification and screening. In the 'high penetrance mutation' setting, I will show that mutation penetrance can vary dramatically with PRS values, with important consequences for genetic testing.

\section{THE MOCOG STUDY: LEARNING FROM EXTRAORDINARY RESPONDERS TO IMPROVE TREATMENT OUTCOMES FOR WOMEN WITH OVARIAN CANCER}

$\underline{\text { Anna de Fazio }}^{1,2,3}$, Dale W. Garsed ${ }^{4,5}$, Ahwan Pandey ${ }^{4}$, Sian Fereday $^{4,5}$, Kathryn Alsop ${ }^{4,5}$, Maartje C. A. Wouters ${ }^{6}$, Bronwyn Gibson-Wright ${ }^{6}$, Flurina Saner ${ }^{4}$, Jessica A. Beach ${ }^{4}$, Katy Milne ${ }^{6}$, Catherine J. Kennedy ${ }^{1,2}$, Joy Hendley ${ }^{4}$, 
Nadia Traficante ${ }^{4,5}$, for the Australian Ovarian Cancer Study Group $^{4}$, Susan J. Ramus ${ }^{7,8}$, Martin Köbel ${ }^{9}$, Brad H. Nelson ${ }^{6}$, Ellen L. Goode ${ }^{10}$, Celeste L. Pearce ${ }^{11}$, Malcolm C. Pike ${ }^{12}$, David D. L. Bowtell ${ }^{4,5,13,14}$ and for the Multidisciplinary Ovarian Cancer Outcomes Group

${ }^{1}$ Centre for Cancer Research, The Westmead Institute for Medical Research, Sydney, NSW, Australia; ${ }^{2}$ Department of Gynaecological Oncology, Westmead Hospital, Westmead, NSW, Australia; ${ }^{3}$ The University of Sydney, Sydney, NSW, Australia; ${ }^{4}$ Peter MacCallum Cancer Centre, Melbourne, Vic, Australia; ${ }^{5}$ Sir Peter MacCallum Department of Oncology, The University of Melbourne, Vic, Australia; ${ }^{6}$ Deeley Research Centre, British Columbia Cancer Agency, Victoria, British Columbia, Canada; ${ }^{7}$ School of Women's and Children's Health, University of NSW, Sydney, Australia; ${ }^{8}$ Garvan Institute of Medical Research, Sydney, NSW, Australia; ${ }^{9}$ Department of Pathology and Laboratory Medicine, Foothill Medical Center, University of Calgary, Calgary, Canada; ${ }^{10}$ Department of Health Science Research, Division of Epidemiology, Mayo Clinic, Rochester, MN, USA; ${ }^{11}$ Department of Epidemiology, University of Michigan School of Public Health, Ann Arbor, MI, USA; ${ }^{12}$ Department of Epidemiology and Biostatistics,

Memorial Sloan Kettering Cancer Center, New York, NY, USA; ${ }^{13}$ Department of Pathology, The University of Melbourne, Vic, Australia; and ${ }^{14}$ Department of Biochemistry and Molecular Biology, The University of Melbourne, Vic, Australia

Background: Most women with high-grade serous ovarian cancer (HGSC) have a poor prognosis and relapse with progressive disease, often within two years of completing primary treatment. However, some women have an extraordinary treatment response and survive more than ten years. We aim to identify factors that influence exceptionally survival, to provide insights that will improve outcomes overall.

Methods: Genomic, immunological and epidemiological determinants of exceptional survival are being investigated in a comprehensive international cohort comprising patients from $>20$ studies across 6 countries. Included patients were diagnosed with advanced stage HGSC with >10-year survival (LTS, longterm survivors), 5-7.99 year survival (moderate-term survivors) and 2-4.99 year survival (short-term survivors), matched for potential confounders.

Results: Whole genome sequencing on 55 LTS so far, shows that somatic mutation burden was higher in LTS. Inactivation of $R B 1$ was significantly enriched in LTS and associated with increased immune-infiltrate. Furthermore, co-occurrence of germline $B R C A$ mutations and RB1 loss was associated with longer survival compared to patients with retained RB1 expression and no germline $B R C A$ mutation.

Conclusions: MOCOG is delineating the full genomic landscape associated with long-term survival in HGSC, and findings to date indicate that specific mutations are associated with enhanced host immune responses and long-term survival.

\section{GENOMICS AND THERAPEUTICS}

Munir Pirmohamed

MRC Centre for Drug Safety Science and Wolfson Centre for Personalised Medicine, The University of Liverpool, Liverpool, $U K$
A key issue for the genomic revolution is how it will help in improving treatment for diseases. As the number of people globally who have their genomes sequenced increases, the depth and breadth of information available will allow us to progress personalised or precision medicine, to ensure that patients get the right treatment at the right dose and at the right time. This will be crucial in ensuring that we optimise the benefit-risk ratio of all therapies (new and old) maximising efficacy and minimising safety. In some cases, genomic information will be directly relevant to the development of new therapies - this is an appropriate strategy given that drugs which are developed on the basis of genetic information seem to have a higher success rate to go to market. Furthermore, we also need to consider how we can use genomics to help in stratifying early phase trials so that not only the correct drug, but also the correct dose can be identified. Genomic information can also provide insights that may lead to re-purposing of medicines for diseases for which there is unmet medical need. Genomic information may also be important in improving the use of drugs which have been available over many years to (a) change the intensity of therapy; and (b) improve the benefit-risk profile. In summary, the genomic revolution offers many opportunities for therapeutics - the key issue will be to develop the evidence base which allows the genomic findings to be translated into patient care.

\section{SIGNALLING IN HIGH-RISK PAEDIATRIC CANCERS - GENOMIC INSIGHTS DRIVING THERAPEUTIC POSSIBILITIES}

$\underline{\text { Paul Ekert and the ZERO Childhood Cancer Project }}$ Translational Tumour Biology, Division of Personalised Medicine, Children's Cancer Institute, Randwick, NSW, Australia; Peter MacCallum Cancer Centre, Parkville, Vic, Australia; and Translational Tumour Biology, Murdoch Children's Research Institute, Parkville, Vic, Australia

Comprehensive genomic analyses of paediatric cancers has driven a rapid expansion of knowledge about the molecular drivers in these high-risk malignancies. The National Zero Childhood Cancer (ZERO) program aims to assess the feasibility of a translating genomic analyses into clinical recommendations. ZERO combines whole genome and transcriptome sequencing, DNA methylation profiling for CNS tumours and sarcomas, and until recently, a cancer associated gene panel. Where possible, in vitro drug screening and patient-derived xenograft drug efficacy testing is attempted. Recommendations are made - tiered by the strength of supporting evidence - through a national Multidisciplinary Tumour Board.

The ZERO national trial (PRISM) is open at all 8 paediatric cancer centres in Australia. An emerging theme is that the combination of WGS and RNA-Seq provide orthogonal data that facilitate the identification of oncogenic driver events beyond the capacity of either approach alone. This includes the curation of splice-site mutations, imbalance in the expression of mutant alleles, the consequences of copynumber aberrations. Another key theme of PRISM is that highrisk paediatric cancers harbour novel, suspected oncogenic drivers for which no biology is currently known. Modelling these events and the responses to therapy are a high priority for the ZERO program. 